\title{
A nonlinear integral operator encountered in the bandwidth sharing of a star-shaped network*
}

\author{
Guy Fayolle ${ }^{\dagger} \quad$ Jean-Marc Lasgouttes*
}

\begin{abstract}
We consider a symmetrical star-shaped network, in which bandwidth is shared among the active connections according to the "min" policy. Starting from a chaos propagation hypothesis, valid when the system is large enough, one can write equilibrium equations for an arbitrary link of the network. This paper describes an approach based on functional analysis of nonlinear integral operators, which allows to characterize quantitatively the behaviour of the network under heavy load conditions.
\end{abstract}

\section{Model description}

Consider a network comprising $N$ links, where several data sources establish connections along routes going through these links. The main concern here is about the policies that can be used to share the bandwidth of the links between active connections, and the effect of these policies on the dynamics of the network.

In this paper, the network is star-shaped (see Figure 1) and all routes are of length 2 , which is a reasonable model for a router. Each star branch contains two links ("in" and "out") and each route is isomorphic to a pair of links.

Let $r=(i, j)$ denote a route on links $i$ and $j$, and $\mathcal{R}$ be the set of all possible routes (with cardinal $N^{2} / 2$ ). Connections are created on $r$ according to a Poisson process with intensity $2 \lambda / N$, so that the total arrival intensity on each link is $\lambda$. A connection lasts until it has transmitted over the network its data, the volume of which follows an exponential law with mean $v$. Each link $i, 1 \leq i \leq N$, has a bandwidth equal to 1 and its load is $\rho \stackrel{\text { def }}{=} \lambda v$.

The state of the system at time $t \in \mathbb{R}$ is given by the number of active connections on each route $\left(c_{r}^{(N)}(t), r \in \mathcal{R}\right) \stackrel{\text { def }}{=} X^{(N)}(t)$. The vector $X^{(N)}(t)$ is in general Markovian and

$$
X_{i}^{(N)}(t) \stackrel{\text { def }}{=} \sum_{r \ni i} c_{r}^{(N)}(t)
$$

*This work has been partly supported by a grant from the Centre National d'Études en Télécommunications.

$\dagger$ INRIA - Domaine de Voluceau BP 105 - Rocquencourt 78153 Le Chesnay cedex

E-mail: Guy.Fayolle@inria.fr, Jean-Marc.Lasgouttes@inria.fr 


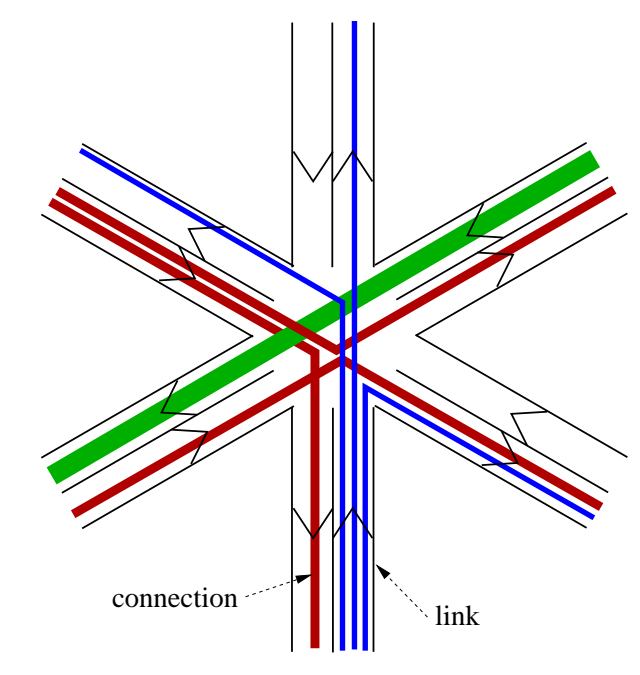

Figure 1: The star-shaped network

is the total number of active connections on link $i, \forall 1 \leq i \leq N$.

It is now necessary to describe how bandwidth allocation is achieved. The "max-min fairness" policy, popular in telecommunication models, being too difficult to be studied rigorously, this article focuses on the "min" policy, proposed by L. Massoullié and J. Roberts [5], in which a connection on $(i, j)$ gets bandwidth

$$
\min \left(\frac{1}{X_{i}^{(N)}(t)}, \frac{1}{X_{j}^{(N)}(t)}\right)
$$

This allocation clearly satisfies the capacity constraints of the system, and can be shown to be sub-optimal with respect to max-min fairness. Its invariant measure equations are however too complicated to be solved explicitly. An efficient method in such situations is to study the model in so-called thermodynamical limit, using mean field analysis.

In order to study the stationary behaviour of the network as $N \rightarrow \infty$, the idea is to assume the conditions of chaos propagation, under which any finite number of links tend to become mutually independent. In this paper, this hypothesis will be considered as a heuristic, to be proved in further studies. Some rigorous studies of this type can be found in Vvedenskaya et al. [8] and Delcoigne et Fayolle [2].

From now on, $\rho<1$ and the system is assumed to be in stationary state $X=\left(c_{r}, r \in \mathcal{R}\right)$. For all $k \geq 0$, the empirical measure of the number of links with $k$ connections is

$$
\alpha_{k}^{(N)} \stackrel{\text { def }}{=} \frac{1}{N} \sum_{1 \leq i \leq N} \mathbb{1}_{\left\{X_{i}^{(N)}=k\right\}}
$$


Symmetry considerations imply that, for all $i \leq N, \mathbb{P}\left(X_{i}^{(N)}=k\right)=\mathbb{E} \alpha_{k}^{(N)}$. Besides, from the chaos propagation hypothesis, a law of large numbers is assumed to hold for $\alpha_{k}^{(N)}$ :

$$
\alpha_{k} \stackrel{\text { def }}{=} \lim _{N \rightarrow \infty} \alpha_{k}^{(N)}=\lim _{N \rightarrow \infty} \mathbb{P}\left(X_{i}^{(N)}=k\right) \stackrel{\text { def }}{=} \mathbb{P}(X=k)
$$

The $\alpha_{k}^{(N)}$ 's, traditionally named mean field, drives the dynamics of the system. The following notation will also prove useful:

$$
\bar{\alpha}^{(N)} \stackrel{\text { def }}{=} \mathbb{E} X_{i}^{(N)}=\sum_{k>0} k \alpha_{k}^{(N)}, \quad \bar{\alpha} \stackrel{\text { def }}{=} \sum_{k>0} k \alpha_{k} .
$$

A heuristic computation (detailed in [3]), yields the following equations:

$$
\begin{aligned}
u_{k} & =\sum_{\ell>0}(k \wedge \ell) \alpha_{l}, \\
\alpha_{k+1} u_{k+1} & =\rho \bar{\alpha} \alpha_{k}, \quad \forall k \geq 0 .
\end{aligned}
$$

While (1.2) resembles a "birth and death process" equation, it is in fact highly non-linear, due to the form of $u_{k}$ and of $\bar{\alpha}$.

The purpose of this paper is to show how the asymptotic behaviour of the system (as $\rho \rightarrow 1$ ) can be derived from the analytical study of the generating functions built from (1.2). This work is a part of the wider study [3], which also gives ergodicity conditions for any topology under the min and max-min policies, shows how equations like (1.2) are derived (also in the case where routes are longer than 2) and presents comprehensive numerical results.

The main byproduct of Theorem 3.3 , is the following asymptotic expansions, valid in a neighborhood of $\rho=1$.

$$
\begin{aligned}
\bar{\alpha} & \approx \frac{1}{(1-\rho)^{2} A} \\
\lim _{k \rightarrow \infty} \rho^{-k} \alpha_{k} & \approx(1-\rho) B \exp \left[\frac{1}{(1-\rho) A}\right]
\end{aligned}
$$

where $A$ and $B$ are non-negative constants. Moreover, if $c(z, 1)$ and $v(z, 1)$ are the solutions of the system of differential equations (3.5), then $A$ can be written as follows:

$$
A=\int_{0}^{\infty} c(z, 1) d z=\lim _{z \rightarrow \infty} z v^{\prime}(z, 1) \approx 1.30 .
$$

Since this system is numerically highly unstable, it has proven difficult (with the "Livermore stiff ODE" solver from MAPLE) to get a better estimate for $A$.

\section{An integral equation for the generating func- tion}

Let $\mathcal{C}(r)$ (resp. $\mathcal{D}(r)$ ), be the circle (resp. the open disk) of radius $r$ in the complex plane. 
Let $\alpha: z \rightarrow \alpha(z)$ be the generating function, a priori defined for $z$ in the closed unit disk

$$
\alpha(z) \stackrel{\text { def }}{=} \sum_{k \geq 0} \alpha_{k} z^{k} .
$$

Denoting $\alpha^{\prime}$ the derivative of $\alpha,(1.2)$ can be rewritten as

$$
\alpha_{k+1} u_{k+1}=\rho \alpha^{\prime}(1) \alpha_{k}, \quad \forall k \geq 0 .
$$

\section{Lemma 2.1 .}

(a) If (2.1) has a probabilistic solution, then, necessarily $\rho<1$, and

$$
\lim _{k \rightarrow \infty} \alpha_{k} \rho^{-k}=K(\rho),
$$

where $K(\rho)$ is a positive constant, bounded $\forall \rho<1$.

(b) The function $\alpha$ satisfies the nonlinear integral equation

$$
\alpha^{\prime}(1)(1-\rho z) \alpha(z)=\frac{1}{2 i \pi} \int_{\mathcal{C}(r)} \alpha(\omega) \alpha\left(\frac{z}{\omega}\right) \frac{d \omega}{(1-\omega)^{2}},
$$

where $|z|<\rho^{-1}$ et $r$ is an arbitrary positive number, with $1<r<\rho^{-1}$.

Proof. When (1.2) has a probabilistic solution, necessarily $\bar{\alpha}=\lim _{m \rightarrow \infty} u_{m}$. Therefore, $\forall \epsilon>0$, there exists a number $M(\epsilon)>0$, such that

$$
\rho \leq \frac{\alpha_{m+1}}{\alpha_{m}} \leq \frac{\rho}{1-\epsilon}, \quad \forall m \geq M(\epsilon),
$$

which implies that $\rho<1$. Moreover, under the same existence hypothesis, one can write

$$
\alpha_{k} \rho^{-k}=\alpha_{0} \prod_{l \geq 0}^{k} \frac{\bar{\alpha}}{\bar{\alpha}-D_{\ell+1}},
$$

where $D_{\ell}=\sum_{m>\ell}(m-\ell) \alpha_{m}$.

When $\ell \rightarrow \infty$, the convergence of the product in (2.3) is equivalent to the convergence of the series of general term $D_{\ell}$, which holds since

$$
D_{\ell} \leq \alpha_{0} \sum_{m \geq \ell}(m-\ell)(\rho+\epsilon)^{m}, \forall \ell \geq M(\epsilon),
$$

so that $\sum_{\ell} D_{\ell}$ behaves like

$$
\sum_{\ell \geq 0} \sum_{n \geq 0} n(\rho+\epsilon)^{\ell+n}=\frac{\rho+\epsilon}{(1-\rho-\epsilon)^{3}} .
$$

Point (a) of the lemma is proven. Point (b) is an application of (a) and of an integral representation used by Hadamard, recalled below (see e.g. [7]). 
Let $a$ and $b$ be functions

$$
a(z)=\sum_{n \geq 0} a_{n} z^{n}, \quad b(z)=\sum_{n \geq 0} b_{n} z^{n},
$$

analytic in the respective disks $\mathcal{D}(R)$ and $\mathcal{D}\left(R^{\prime}\right)$. Then the function

$$
c(z)=\sum_{n} a_{n} b_{n} z^{n}
$$

has a radius of convergence greater than $R R^{\prime}$ and has the integral form

$$
c(z)=\frac{1}{2 i \pi} \int_{\mathcal{L}} a(\omega) b\left(\frac{z}{\omega}\right) \frac{d \omega}{\omega},
$$

where $\mathcal{L}$ is a closed contour containing the origin, and on which $|\omega|<$ $R,\left|\frac{z}{\omega}\right|<R^{\prime}$.

From (a), $\alpha$ has a radius of convergence at least equal to $\rho^{-1}$ : this property, used in Hadamard's formula, leads directly to (2.2).

The following Lemma provides a finer description of $\alpha(z)$.

Lemma 2.2. The function $\alpha$ is meromorphic and can be written as

$$
\alpha(z)=\sum_{i=1}^{\infty} \frac{a_{i}}{\rho^{-i}-z} .
$$

Moreover, for any sequence of circles $\mathcal{C}\left(R_{n}\right)$, such that

$$
(1+\epsilon) \rho^{-n} \leq R_{n} \leq(1-\epsilon) \rho^{-(n+1)}, \quad 0<\epsilon<\frac{1-\rho}{1+\rho},
$$

one has $\left|\alpha\left(R_{n}\right)\right|=o(1)$ as $n \rightarrow \infty$.

Proof. The integral equation (2.2) allows for the analytic continuation of $\alpha$ in the whole complex plane. Indeed, from point (a) of Lemma 2.1 $\alpha$ is holomorphic in $\mathcal{D}\left(\rho^{-1}\right)$ and its first singularity is a simple pole at the point $z=\rho^{-1}$. An application of Cauchy's theorem to the integral in (2.2) leads to

$$
\frac{1}{2 i \pi} \int_{\mathcal{C}\left(R_{1}\right)}=\frac{1}{2 i \pi} \int_{\mathcal{C}(r)}+\operatorname{Residue}\left(\rho^{-1}\right)
$$

where $R_{1}$ is defined above. Since $\alpha(\omega)$ and $\alpha(z / \omega)$ are analytic in the regions

$$
|\omega|<\rho^{-1}, \quad\left|\frac{z}{\omega}\right|<\rho^{-1},
$$

the integral is a function of $z$ analytic in the ring-shaped area $\rho^{-1}<|z|<\rho^{-2}$. The same holds for $\alpha$, thanks to the left-hand side of (2.2). Equation (2.4) follows by recurrence. 
The second part of the lemma is obtained by application of Cauchy's theorem to the integral in (2.2) along the $\operatorname{circle} \mathcal{C}\left(R_{n}\right)$ :

$$
\alpha^{\prime}(1)(1-\rho z) \alpha(z)-\sum_{i=1}^{n} \frac{a_{i} \alpha\left(z \rho^{i}\right)}{\left(\rho^{-i}-1\right)^{2}}=\int_{\mathcal{C}\left(R_{n}\right)} \alpha(\omega) \alpha\left(\frac{z}{\omega}\right) \frac{d \omega}{(1-\omega)^{2}} .
$$

One can make the analytic continuation of the above equality, the left-hand side of which is analytic in $|z| \leq R_{n}$, letting $z$ reach the circle $\mathcal{C}\left(R_{n}\right)$ along a simple curve which avoids the polar singularities $\rho^{-i}, i=1, \ldots, n$. Bounding the modulus of the integral yields the inequality

$$
\left(\rho R_{n}-1\right) \mathcal{M}\left(R_{n}\right) \leq A_{n} \sup _{i \leq n-1}\left(\mathcal{M}\left(R_{i}\right)\right)+D \mathcal{M}\left(R_{n}\right) \int_{\mathcal{C}\left(R_{n}\right)} \frac{|d \omega|}{|1-\omega|^{2}}
$$

where $D$ is a positive constant and $A_{n}$ is bounded $\forall n \leq \infty$. By induction, $\lim _{n \rightarrow \infty} \mathcal{M}\left(R_{n}\right)=0$ follows easily, and the proof is completed.

Combining (2.4) with (2.2), a straightforward computation of residues gives

$$
\alpha^{\prime}(1)(1-\rho z) \sum_{i=1}^{\infty} \frac{a_{i}}{\rho^{-i}-z}=\sum_{i, j=1}^{\infty} \frac{a_{i} a_{j} \rho^{-j}}{\left(\rho^{-(i+j)}-z\right)\left(1-\rho^{-j}\right)^{2}} .
$$

Let $a: z \rightarrow a(z)$ be the generating function

$$
a(z) \stackrel{\text { def }}{=} \sum_{k \geq 0} a_{k+1} z^{k}
$$

defined for $z$ in a bounded domain of the complex plane, including the origin. Using point (a) of Lemma 2.1 and (2.4), the following relations hold

$$
\left\{\begin{array}{l}
a_{1}=\rho K(\rho) \\
\alpha_{k-1}=\rho^{k} a\left(\rho^{k}\right), \quad \forall k \geq 1
\end{array}\right.
$$

Since $\alpha_{0}<1-\rho$, the function $a$ is thus analytic in the disk $\mathcal{D}(\rho)$. Identifying the coefficients of the power series in $z$ in (2.5), one gets

$$
\alpha^{\prime}(1)\left(\rho^{k-1}-1\right) a_{k}=\sum_{j=1}^{k-1} \frac{a_{j} a_{k-j} \rho^{j+k-1}}{\left(1-\rho^{j}\right)^{2}}, \quad \forall k \geq 1 .
$$

It follows easily by recurrence that the $a_{i}$ 's are of alternate signs, with $a_{1}>0$.

Let

$$
f(t) \stackrel{\text { def }}{=} \sum_{j=1}^{\infty}\left(\frac{\rho^{j}}{1-\rho^{j}}\right)^{2} t^{j}=t \sum_{j=1}^{\infty} \frac{j \rho^{j+1}}{1-t \rho^{j+1}}, \quad \rho<1,|t|<\rho^{-2} .
$$


Hadamard's formula, when applied in (2.7), implies the integro-differential equation

$$
\alpha^{\prime}(1)[a(\rho z)-a(z)]=\frac{a(\rho z)}{2 i \pi} \int_{\mathcal{C}(r)} a(\omega) f\left(\frac{z}{\omega}\right) d \omega,
$$

valid in the domain $\left\{r \leq \rho,|z|<\rho^{-2}\right\}$.

Taking the second form for $f$ in (2.8), which in fact converges in the domain $\{\rho<1, \Re(z) \leq 0\}$, and applying Cauchy's theorem to the integral in (2.9), one obtains the functional equation

$$
\alpha^{\prime}(1)[a(\rho z)-a(z)]=a(\rho z) b(z), \quad|z| \leq \rho, \rho<1,
$$

where

$$
b(z) \stackrel{\text { def }}{=} z \sum_{j=1}^{\infty} j \rho^{j+1} a\left(\rho^{j+1} z\right) .
$$

From (2.10), let's make now the analytic continuation of $a$ in the nested disks $\mathcal{D}\left(\rho^{-n}\right), n \geq 1$. It appears that $a$ has no singularity at finite distance, and consequently is an integral function. From the general theory [7, it follows that $a$ is completely characterized by its zeros and its order at infinity.

Let $z_{0}$ be an arbitrary zero of $a$. From (2.10) again, $a\left(\rho^{-i} z_{0}\right)=0$ and the zeros of $a$ form families of points in geometric progression with parameter $\rho^{-1}$. It suffices to determine the zeros of smallest modulus, but, alas they do not have any explicit form and numerical schemes are highly unstable. However, from (2.6), $b(1)=\alpha^{\prime}(1)$, so that (2.10) implies $a(1)=0$ together with

$$
a\left(\rho^{-i}\right)=0, \quad \forall i \geq 0 .
$$

\section{On the asymptotic behaviour around $\rho=1$}

In order to assess the practical value of the "min" policy, it is important to evaluate the system behaviour in heavy traffic conditions. The numerical calculations in [3] show that the distribution of any $X_{i}$ is modal, which is not common in known models.

In this section, it will be convenient to consider $\rho$ not only as a parameter but as a plain variable. Therefore, in all quantities of interest, $\rho$ will appear as an explicit variable, e.g. $a(z, \rho), f(z, \rho)$ or $a_{k}(\rho)$.

The fundamental ideas of the analysis will be given after the next lemma, which proposes a scaling - likely to be the only interesting one - for the function $a(z, \rho)$.

Let

$$
\left\{\begin{array}{l}
\xi(\rho) \stackrel{\text { def }}{=} \frac{-a_{1}(\rho)}{(\log \rho)^{3} \alpha^{\prime}(1, \rho)}, \\
c_{k}(\rho) \stackrel{\text { def }}{=} \frac{-a_{k}(\rho)}{(\log \rho)^{3} \alpha^{\prime}(1, \rho) \xi(\rho)^{k}}, \quad \forall k \geq 1, \\
c(z, \rho) \stackrel{\text { def }}{=} \sum_{i=0}^{\infty} c_{k+1}(\rho) z^{k} .
\end{array}\right.
$$


The reader will easily convince himself that the factor $(1-\rho)^{3}$ arises rather naturally; however the factor $-\log ^{3} \rho$ has been chosen here, since it provides more compact formulas in the forthcoming results.

Lemma 3.1. Let

$$
v(z, \rho) \stackrel{\text { def }}{=} \frac{(\log \rho)^{2}}{2 i \pi} \int_{\mathcal{C}(1)} c(\omega, \rho) f\left(\frac{z}{\omega}, \rho\right) d \omega, \quad|z|<\rho^{-2} .
$$

The following functional relations hold:

$$
\left\{\begin{aligned}
a(z, \rho) & =a_{1}(\rho) c(z \xi(\rho), \rho) \\
c(0, \rho)= & 1 \\
c(z, \rho) & =c(\rho z, \rho)[1+\log \rho v(z, \rho)] \\
& =c(\rho z, \rho)\left[1+z(\log \rho)^{3} \sum_{i=1}^{\infty} i \rho^{i+1} c\left(\rho^{i+1} z, \rho\right)\right] .
\end{aligned}\right.
$$

The coefficients $c_{k}(\rho), k \geq 1$, are of alternate signs and the function $c(z, \rho)$ has the following properties.

(a) there exists only one solution $c(z, \rho)$ of (3.2), which is integral with respect to $z$ and bi-analytic in $(z, \rho)$ in the region $0<\rho<1$.

(b) Define

$$
g(t) \stackrel{\text { def }}{=} \sum_{j=1}^{\infty} \frac{t^{j}}{j^{2}}=\int_{0}^{t} \frac{-\log (1-u) d u}{u}, \quad \forall|t| \leq 1,
$$

denoted by some authors as $\operatorname{dilog}(1-t)$. Then $c(z, 1)$ exists and satisfies the integro-differential equation

$$
z \frac{\partial c(z, 1)}{\partial z}=-\frac{c(z, 1)}{2 i \pi} \int_{\mathcal{C}(r)} c(\omega, 1) g\left(\frac{z}{\omega}\right) d \omega, \quad|r|=1, \quad \forall|z| \leq 1,
$$

which rewrites in the form

$$
z \frac{\partial c(z, 1)}{\partial z}=-c(z, 1) \int_{0}^{z} c(\omega, 1) \log \left(\frac{z}{\omega}\right) d \omega
$$

which is equivalent to the non-linear differential system

$$
\left\{\begin{array}{l}
z \frac{\partial c(z, 1)}{\partial z}+c(z, 1) v(z, 1)=0 \\
z \frac{\partial^{2} v(z, 1)}{\partial z^{2}}+\frac{\partial v(z, 1)}{\partial z}=c(z, 1)
\end{array}\right.
$$

with initial conditions

$$
v(0,1)=0, \quad \frac{\partial v(z, 1)}{\partial z}_{\mid z=0}=1, \quad c(0,1)=1 .
$$

(c) Moreover, $c(z, 1)$ is analytic in the open complex plane, except at a negative real point $q$, and $c(z, 1) \neq 0, \forall z \neq q \cup \infty$. 
Proof. The first three equations in (3.2) follow directly from the definition (2.7) and (2.10) of the coefficients $a_{k}(\rho)$, the fourth one coming from the analytic continuation of (2.9). Existence and uniqueness are simple consequences of the convolution equation (2.7).

The properties relative to the morphology of $c(z, \rho), \rho \leq 1$, are more intricate. First, the reader will notice that (3.3) can be obtained rigorously from (2.7) or (2.8), but not from (3.2)! Then, there is a phase transition when $\rho=1$. We will return to this topic in Section 4 .

It is interesting to note that the function $w(y) \stackrel{\text { def }}{=} v\left(e^{y}\right)+1$ satisfies the so-called Blasius [1] third-order differential equation

$$
w^{\prime \prime \prime}(y)+w(y) w^{\prime \prime}(y)=0,
$$

which arises in hydrodynamics to describe the stationary evolution of a laminar boundary layer along a flat plate! The explicit solution of this equation is still unknown, albeit it has been studied by many authors over the last decades (see e.g. [6]).

Starting from Lemma 3.1, it is now possible to sketch the main ideas of the proposed method. The Gordian knot amounts to the evaluation of $\xi(\rho)$, defined in (3.1). This can be done via the anchoring equation

$$
c(\xi(\rho), \rho)=0,
$$

which follows from 2.11 and from the first equation of (3.2). From the structure of the third equation of (3.2), it appears that the smallest positive solution of $c(u, \rho)=0$ satisfies

$$
\frac{-1}{u \log \rho}=(\log \rho)^{2} \sum_{i \geq 1} i \rho^{i+1} c\left(u \rho^{i+1}, \rho\right),
$$

the right-hand side of which is an analytic function, bounded in any compact set $\forall \rho \leq 1$ : as $\rho \rightarrow 1$, necessarily $u \rightarrow \infty$ and all positive zeros of the anchoring equation are sent to infinity. The key is to find the asymptotic behaviour in $z$ of the various functions, in the cone $0 \leq z \leq \mathcal{U}(\rho)$, which contains $\xi(\rho)$ : in this cone, $c(z, \rho)$ is close to $c(z, 1)$-in some sense - and

$$
v(z, \rho) \approx w(z, \rho) \stackrel{\text { def }}{=} \log ^{2} \rho \sum_{i \geq 1} i \rho^{i+1} c\left(z \rho^{i+1}, 1\right) .
$$

Since $w(z, 1)$ has a logarithmic behaviour, $\xi(\rho)$ can be obtained by direct inversion. The sketch of the proof is outlined below.

We will need the Mellin transform (see e.g. 4]) of $c(z, 1)$, defined as

$$
c^{*}(s) \stackrel{\text { def }}{=} \int_{0}^{\infty} x^{s-1} c(x) d x .
$$

The behaviour of $c(z, 1)$ in the region $\Re(z)>0$, given in Section 4 implies the existence of $c^{*}(s, 1), \forall s, \Re(s)>0$ and of all the moments of $c(z, 1)$ on the positive real axis. 
Lemma 3.2. The function $w(z, \rho)$ defined by (3.7) admits, $\forall z, \Re(z)>0$, the asymptotic expansion

$$
\begin{aligned}
w(z, \rho) & =c^{*}(1,1) \log (\rho z)-\frac{\partial c^{*}(1,1)}{\partial s} \\
& +(\log \rho)^{2}[(\log z) \Phi(z, \rho)+\Psi(z, \rho)]+\mathcal{O}\left(z^{-d}\right),
\end{aligned}
$$

where $d$ is an arbitrary positive number, $\Phi$ and $\Psi$ are fluctuating functions of small amplitude for $\rho \neq 1$, which vanish for $\rho=1$.

Proof. From the Mellin transform inversion formulas,

$$
w(z, \rho)=\frac{1}{2 i \pi} \int_{\sigma-i \infty}^{\sigma+i \infty} z^{-s} c^{*}(s+1,1)\left[\frac{\log \rho}{1-\rho^{s}}\right]^{2} d s, \quad \forall \sigma \in(-1,0) .
$$

Let $s_{n} \stackrel{\text { def }}{=} 2 i n \pi \log ^{-1} \rho, \forall n \in$ Z. Cauchy's theorem can be applied to (3.9), by integrating along the vertical line $\Re(s)=d>0$, so that

$$
w(z, \rho)=\sum_{n \in \mathbf{Z}} z^{-s_{n}}\left[c^{*}\left(s_{n}+1,1\right) \log (\rho z)-\frac{\partial c^{*}\left(s_{n}+1,1\right)}{\partial s}\right]+\mathcal{O}\left(z^{-d}\right) .
$$

The series above is equal to the sum of the residues, taken on the vertical line $\Re(s)=0$, and is uniformly bounded, $\forall \rho \leq 1$. Indeed, an integration by parts gives the inequality

$$
\left|c^{*}\left(s_{n}+1,1\right)\right|=\left|\int_{0}^{\infty} y^{s_{n}} c(y, 1) d y\right| \leq\left|\frac{\Gamma\left(s_{n}+1\right)}{\Gamma\left(s_{n}+k+1\right)}\right| \int_{0}^{\infty} y^{k}\left|c^{(k)}(y, 1)\right| d y,
$$

where $c^{(k)}$ is the $k$-th derivative of $c(z, 1)$ and $\Gamma(x)$ is the usual Eulerian function. Since

$$
\left|\frac{\Gamma\left(s_{n}+1\right)}{\Gamma\left(s_{n}+k+1\right)}\right|<\frac{\log ^{k} \rho}{k !},
$$

the proof of (3.8) and of the lemma is concluded.

Theorem 3.3. For some real number $d>1$, the following expansions hold.

$$
\begin{aligned}
\rho \xi(\rho) & =\exp \left[-\frac{1}{\log \rho c^{*}(1,1)}-\frac{\partial \log c^{*}(1,1)}{\partial s}\right]\left(1+\mathcal{O}\left((\log \rho)^{d}\right)\right) \\
\alpha^{\prime}(1, \rho) & =\frac{1}{(\log \rho)^{2} c^{*}(1,1)}+\mathcal{O}\left((\log \rho)^{d}\right)
\end{aligned}
$$

Proof. From (3.6), $\xi(\rho)$ is solution of the equation in $x$

$$
1+\log \rho v(x, \rho)=0 .
$$

A deep analysis, which is not included here, shows that this equation can be replaced by the locally equivalent equation

$$
1+\log \rho w(x, \rho)+\mathcal{O}\left((\log \rho)^{p}\right)=0,
$$


where $p$ is a positive number, $p>1$. Then, from Lemma 3.2 ,

$$
-\frac{1}{\log \rho}=\log (\rho x) c^{*}(1,1)-\frac{\partial c^{*}(1,1)}{\partial s}+\log (\rho x) \Phi(x, \rho)+\Psi(x, \rho)+\mathcal{O}\left(x^{-d}\right),
$$

which implies (3.11).

For the computation of $\alpha^{\prime}(1, \rho)$, one uses the simple relation

$$
\rho \alpha^{\prime}(1, \rho)=\frac{\alpha_{1}}{\alpha_{0}}-\alpha_{1},
$$

obtained by derivation of (2.2) at $z=0$, and (3.12) follows.

\section{Remarks and complements}

It is important to note that one of the main technical difficulties of the problem, besides its strongly non-linear feature, comes from the phase transition which appears for $\rho=1$. Actually, $c(z, \rho)>0$ for $0 \leq z<\xi(\rho)$ and $c(\xi(\rho), \rho)=0$. Then, for $z \gg \xi(\rho), c(z, \rho)$ has wild unbounded oscillations. In particular, this implies that $\int_{0}^{\infty} c(x, \rho) d x$ does not exist.

On the other hand, when $\rho=1$, the $c(z, 1)$ is no more an integral function: it has a singularity (which seems to be a pole of order 3 ) located on the negative real axis; it does not vanish for $z \geq 0$, and the quantity $\int_{0}^{\infty} c(x, 1) d x=A$ is finite. In the half plane $\Re(z)>0$, the following expansions hold:

$$
\begin{aligned}
& c(z, 1)=\exp \left[-\frac{c^{*}(1,1)}{2} \log ^{2} z+B \log z+\frac{D \log z}{z^{2}}+o\left(\frac{\log z}{z^{2}}\right)\right], \\
& v(z, 1)=c^{*}(1,1) \log z+B+\frac{D \log z}{z^{2}}+o\left(\frac{\log z}{z^{3}}\right),
\end{aligned}
$$

where $B$ and $D$ are some constants.

Finally, iterating (3.2), one could improve some of the estimates given in the previous section, rewriting $c(z, \rho)$ as

$$
c(z, \rho)=c\left(z \rho^{I+1}, \rho\right) \prod_{i=0}^{I}\left[1+\log \rho v\left(z \rho^{i}, \rho\right)\right],
$$

where $I$ is an arbitrary positive integer. The above product is uniformly convergent, $\forall I \leq \infty$, for all $z$ in a compact set of the complex plane, since it behaves like the series

$$
z(\log \rho)^{3} \sum_{k \geq 0} \sum_{i \geq 1} i \rho^{i+k+1} c\left(z \rho^{i+k+1}, \rho\right) .
$$

This series has its modulus bounded by $\left|z \rho^{2} c(\rho z, \rho)\right|$, so that, from the maximum modulus principle, it converges uniformly. Then, one can choose $I$ to ensure

$$
0<z \rho^{I} \leq 1 \text {, i.e. } I \leq-\frac{\log z}{\log \rho},
$$

and make use of the properties of the $\Gamma$ function to estimate the product. 


\section{References}

[1] Blasius, H. Grenzschichten in flüssigkeiten mit kleiner reibung. Z. Math Phys. 56 (1908), 1-37. English translation in NACA TM 1256.

[2] Delcoigne, F., And Fayolle, G. Thermodynamical limit and propagation of chaos in polling systems. Markov Processes and Related Fields 5, 1 (1999), 89-124.

[3] Deneux, T., Fayolle, G., de la Fortelle, A., and lasgouttes, J.M. Allocation de bande passante dans les grands réseaux : autour de la politique "min". Tech. rep., INRIA, June 2000. to appear.

[4] Doetsch, G. Handbuch der Laplace Transformation, vol. 1-3. BirkhaüserVerlag. Basel, 1955.

[5] Massoulié, L., And Roberts, J. Private communication, 1998.

[6] Schlichting, H. Boundary layer theory. McGraw-Hill Book Co., Inc., New York, 1960. Translated by J. Kestin. 4th ed. McGraw-Hill Series in Mechanical Engineering.

[7] Titchmarsh, E. C. The Theory of Functions, 2nd ed. Oxford University Press, 1939.

[8] Vvedenskaya, N. D., Dobrushin, R. L., and Karpelevich, F. I. A queueing system with a choice of the shorter of two queues - an asymptotic approach. Problems Inform. Transmission 32 (1996), 15-27. 